\title{
Amino Acid Requirements of Strains of Chlamydia trachomatis and C. psittaci Growing in McCoy Cells: Relationship with Clinical Syndrome and Host Origin
}

\author{
By I. AlLAN† AND J. H. PEARCE* \\ Department of Microbiology, University of Birmingham, Birmingham B15 2TT, U.K.
}

(Received 19 October 1982; revised 22 January 1983)

\begin{abstract}
The effects of omission of individual amino acids from growth medium on the multiplication of a range of Chlamydia trachomatis and $C$. psittaci strains in cycloheximide-treated McCoy cells have been assessed. Differences in requirements were revealed which for $C$. trachomatis strains correlated with clinical syndrome and for $C$. psittaci with host origin. All 11 strains of C. trachomatis examined showed a requirement for addition of histidine to the medium; this was not shown by any of four $C$. psittaci strains. Among the strains of $C$. trachomatis, three from cases of trachoma, representing serotypes $\mathrm{A}, \mathrm{B}$ and $\mathrm{C}$, showed a distinctive requirement for the addition of tryptophan to the medium, whilst six strains of oculogenital origin, representing serotypes $\mathrm{D}$ I, exhibited no requirement for tryptophan or methionine; a lymphogranuloma venereum and a 'fast variant' strain both showed a requirement for methionine. Of the four $C$. psittaci strains from different hosts, three showed distinct patterns of amino acid requirements. All chlamydiae required the addition of valine to medium and the majority required leucine, phenylalanine and also glutamine.
\end{abstract}

\section{INTRODUCTION}

The obligate parasitism of the chlamydiae is believed to arise in part from the dependence of these metabolically disabled bacteria on the provision of high-energy intermediates by their host cells (Moulder, 1974). As previously reviewed (Allan \& Pearce, 1983), chlamydiae are also dependent on the cellular environment for certain amino acids and their availability may determine whether dormant or productive infection is expressed in the parasitized cells.

We have reported that growth of Chlamydia psittaci strain guinea pig inclusion conjunctivitis (GP-IC), in cycloheximide-treated McCoy cells appears to be regulated by the extracellular concentration of leucine, phenylalanine and valine (Allan \& Pearce, 1983). The question directly arising from these observations was whether the effects were determined by the host cell or by the parasite. If it was the latter, then different chlamydial strains might be expected to exhibit varied amino acid requirements reflecting the nutritional environments in which they had evolved.

To test this possibility, we have examined a range of $C$. trachomatis and $C$. psittaci strains and determined the effects of omission of single amino acids on growth in McCoy cells. Chlamydia psittaci strains show a varied host and tissue range. Chlamydia trachomatis strains are primarily pathogens of man, and epidemiological studies have largely segregated the known serotypes into groups associated with the chronic eye disease, trachoma (serotypes $\mathrm{A}, \mathrm{B}, \mathrm{Ba}, \mathrm{C}$ ), genital disease

† Present address: Department of Microbiology and Immunology, UCLA School of Medicine, Center for Health Sciences, Los Angeles, California 90024, U.S.A.

\footnotetext{
Abbreviations: BSS, balanced salts solution; FBS, foetal bovine serum; GP-IC, guinea pig inclusion conjunctivitis strain of $C$. psittaci; i.f.u., inclusion-forming units; p.i., post-infection.
} 
and inclusion conjunctivitis (serotypes $\mathbf{D}-\mathrm{K}$ ), and the sexually transmitted disease, lymphogranuloma venereum ( $\left.L G V ; L_{1}, L_{2}, L_{3}\right)$.

Here we report that the amino acid requirements of strains of C. trachomatis and C. psittaci appear to show a correlation with clinical syndrome and host origin, respectively.

\section{METHODS}

Organisms. Chlamydiae (listed below) were produced by growth in irradiated McCoy cell monolayers; inoculation and growth medium was as previously described (Allan \& Pearce, 1983). Briefly, at $48 \mathrm{~h}$ post-infection (p.i.) chlamydiae were harvested by sonication of monolayers $\left(70 \mathrm{~W}, 10 \mathrm{~s}, 0^{\circ} \mathrm{C}\right)$ and partially purified by differential centrifugation $\left(500 \mathrm{~g}, 10 \mathrm{~min}, 4^{\circ} \mathrm{C}\right.$, supernatant retained; $24000 \mathrm{~g}, 60 \mathrm{~min}, 4^{\circ} \mathrm{C}$, pellet retained). Organism pellets were resuspended by sonication in sucrose/phosphate/glutamate buffer (Kuo et al., 1977) and samples were stored at $-70^{\circ} \mathrm{C}$.

The following were grown. Chlamydia trachomatis strains: A/SA-1/OT (Wang \& Grayston, 1971a); B/TW5/OT; C/UW-1/OT; D/Cal-8/ON (Treharne et al., 1977); E/MRC-4/IC (Collier \& Smith, 1967); F/MRC-301/U; G/IOL-238/R; H/UW-4/Cx; I/UW-12/U; $\mathrm{L}_{2} / 434 / \mathrm{Bu}$ (Treharne et al., 1977); $\mathrm{L}_{2} / \mathrm{MRC}-4 \mathrm{f} /$ - (Reeve \& Taverne, 1963); the classification is serotype/strain/origin (OT, ocular trachoma; ON, ophthalmia neonatorum; IC, inclusion conjunctivitis; $\mathrm{U}$, urethra; $\mathrm{R}$, rectum; $\mathrm{Cx}$, cervix; $\mathrm{Bu}$, bubo). Chlamydia psittaci strains: enzootic abortion of ewes (EAE; Stamp et al., 1950); feline keratoconjunctivitis (FKC; Darougar et al., 1977); guinea pig inclusion conjunctivitis (GP-IC; Murray, 1964); meningopneumonitis (Cal-10; Francis \& Magill, 1938).

Cell culture and monolayer preparation. This was as described previously (Allan \& Pearce, 1983). McCoy cell cultures at passage levels before and after completion of these experiments were negative for mycoplasmas when tested by co-cultivation with mycoplasma-free Vero cells (Allan \& Pearce, 1983).

Assessment of the effect on chlamydial multiplication of deletion of single amino acids from the growth medium. Suspensions of chlamydiae in Hanks' balanced salts solution (BSS) containing $5 \%(\mathrm{v} / \mathrm{v})$ foetal bovine serum (FBS) sufficient to infect approx. $10 \%$ of cells were centrifuged with McCoy cell monolayers which had been irradiated $1 \mathrm{~d}$ earlier (Griffiths et al., 1976). Subsequently, the inoculum was removed and control or test growth medium added. Monolayers were incubated at $35^{\circ} \mathrm{C}$ in air $/ \mathrm{CO}_{2}(95: 5, \mathrm{v} / \mathrm{v}$ ) for $48 \mathrm{~h}$ prior to fixation (GP-IC and Cal-10 at $26 \mathrm{~h}$ ), Giemsa staining and microscopic assessment of the level of infection as inclusion-forming units (i.f.u.) per monolayer. The preparation of growth media and the examination of the effect of omission of single amino acids on growth were as described in the previous paper (Allan \& Pearce, 1983). Requirements for each strain were analysed in two experiments using separate batches of media for each experiment to minimize possible errors arising from faulty media preparation.

\section{RESULTS}

\section{Selection of strains for analysis of amino acid requirements}

The amino acid requirements of serotypes of $C$. trachomatis largely associated with trachoma (Table 1) and oculogenital infection (Table 2) were examined. A single LGV strain of the most common serotype $\left(\mathbf{L}_{2}\right)$ was also studied (Table 3 ) and its amino acid requirements compared with those of a 'fast' egg-killing strain which, by serological analysis, has been argued to be LGV (serotype $\mathrm{L}_{2}$ ) in origin (Wang \& Grayston, $1971 b$ ).

Current U.K. safety regulations restricted analysis of strains of $C$. psittaci to GP-IC, EAE and Cal-10 (pathogen category C; Howie, 1978) and FKC (pathogen category B); the latter required special containment facilities. GP-IC, EAE and FKC have guinea pig, sheep and cat as their host of origin. The origin of Cal-10 is uncertain: it was isolated from ferrets inoculated with throat washings of a patient suspected of having epidemic influenza. An apparently identical organism was isolated in ferrets from patients with epidemic respiratory disease which did not appear to be influenza; no similar organism could be isolated from normal ferrets (Francis \& Magill, 1938). Whether Cal-10 arose from a natural infection of ferrets or by ferret selection from what might have been psittacosis caused by an avian strain of C. psittaci remains unclear; it is considered non-pathogenic for man.

\section{Effect of omission of single amino acids on the growth of C. trachomatis strains isolated from cases of trachoma}

The three strains tested were all markedly inhibited when either glutamine, histidine, leucine, phenylalanine, tryptophan or valine was omitted from the growth medium (Table 1). Omission of isoleucine led to varying numbers of infected cells all of which had markedly smaller inclusion 
Table 1. Effect of single amino acid omissions on the growth of trachoma strains of C. trachomatis in cycloheximide-treated monolayers

Chlamydial suspensions in Hanks' BSS supplemented with $5 \%$ FBS were centrifuged with irradiated McCoy cell monolayers and after removal of the inoculum, growth medium containing 12 of the 13 amino acids of Eagle's MEM, $5 \%$ dialysed FBS and $2 \mu \mathrm{g}$ cycloheximide $\mathrm{ml}^{-1}$ was added. Monolayers (48 h p.i.) were fixed, Giemsa-stained and infection assessed microscopically. In control monolayers, which were incubated with complete growth medium, approx. $10 \%$ of cells were infected. The paired values shown are the results for two experiments, each done with separate batches of medium; individual values are means for duplicate monolayers with at least 2500 cells per monolayer scanned for inclusions.

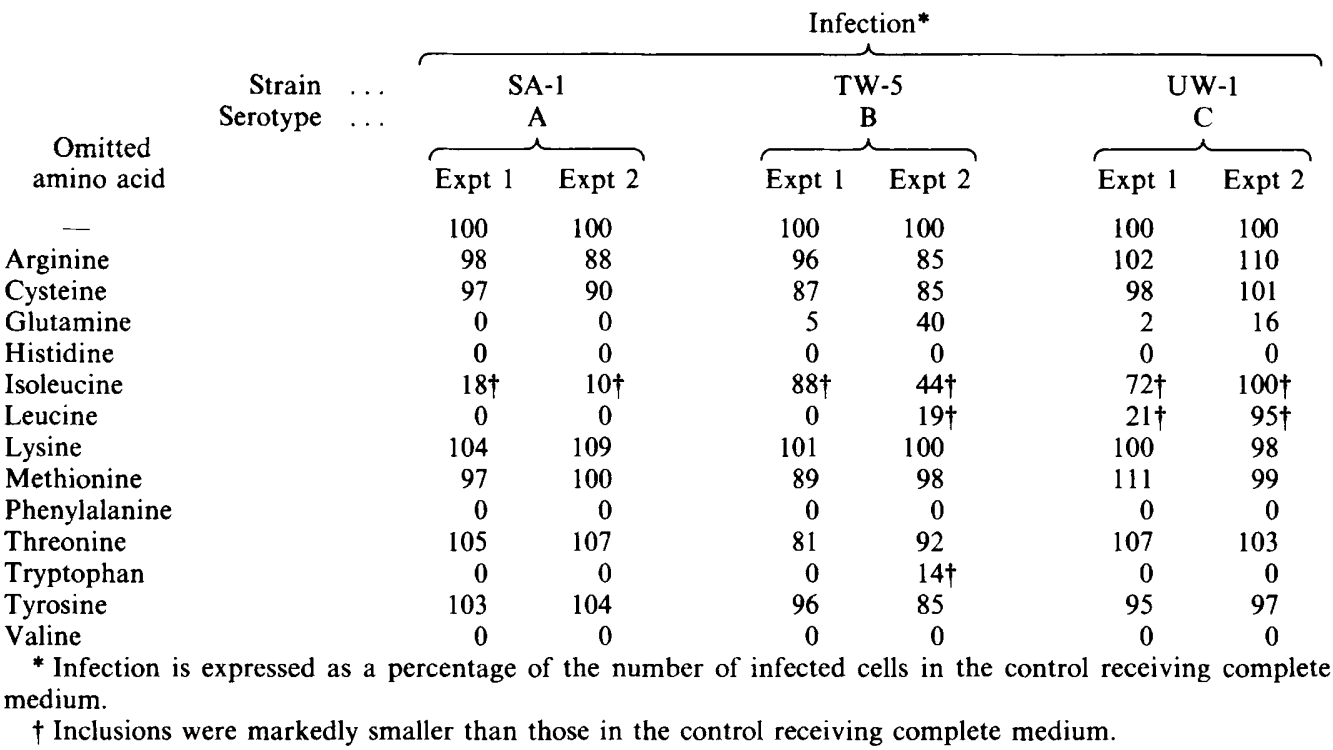

bodies than those produced in cells incubated with fully supplemented medium. The inhibitory effect of tryptophan omission distinguished this group from $C$. trachomatis strains not isolated from cases of trachoma (Tables 2 and 3; see later).

\section{Effect of omission of single amino acids on the growth of $C$. trachomatis strains isolated from genital or ocular infection}

Multiplication of six oculogenital strains of $C$. trachomatis, representing serotypes D-I, was largely abolished when either glutamine, histidine, phenylalanine or valine were omitted from the growth medium; glutamine omission severely curtailed growth (Table 2). Omission of leucine had a variable effect: strains MRC-4 and MRC-301 grew normally in the absence of supplied leucine, whilst strains IOL-238 and UW-12 failed to multiply; Cal-8 and UW-4 produced intermediate numbers of inclusions but these were much smaller than inclusions produced in the fully supplemented control medium.

The oculogenital strains multiplied in the absence of supplied methionine or tryptophan; this distinguished them from strain 434 (LGV) (Table 3) and the trachoma strains, respectively.

Effect of omission of single amino acids on the growth of a C. trachomatis LGV strain and a 'fast' egg-killing strain

Omission of either glutamine, histidine, leucine, methionine, phenylalanine or valine markedly inhibited the growth of both of these strains (Table 3). In the absence of supplied isoleucine a reduced number of small inclusions were produced. The requirement of these two strains for methionine distinguished them from the trachoma and oculogenital strains of $C$. trachomatis examined. But the LGV strains, like the oculogenital strains, differed from the trachoma strains in having no requirement for added tryptophan. 
Table 2. Effect of single amino acid omissions on the growth of oculogenital strains of C. trachomatis in cycloheximide-treated monolayers

Experimental conditions were as described in Table 1.

\begin{tabular}{|c|c|c|c|c|c|c|c|c|c|c|c|c|}
\hline & & & & & & Inf & Ion* & & & & & \\
\hline $\begin{array}{r}\text { Strain } \\
\text { Serotype }\end{array}$ & $\mathrm{C}$ & & $\begin{array}{r}\text { MR } \\
\text { F }\end{array}$ & $C-4$ & MR & -301 & $10 \mathrm{~L}$ & 38 & U & -4 & UV & -12 \\
\hline amino acid & Expt & Expt & 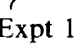 & Expt & & Expt & $x n t$ & Exp & vat & Expt & Expt & Expt 2 \\
\hline - & 100 & 100 & 100 & 100 & 100 & 100 & 100 & 100 & 100 & 100 & 100 & 100 \\
\hline Arginine & 92 & 106 & 100 & 109 & 91 & 96 & 88 & 96 & 104 & 94 & 105 & 105 \\
\hline Cysteine & 88 & 67 & 110 & 110 & 97 & 91 & 80 & 92 & 107 & 96 & 108 & 109 \\
\hline Glutamine & $40 \dagger$ & $39 \dagger$ & $7 \dagger$ & $5 \dagger$ & $11 \dagger$ & $10 \dagger$ & $47 \dagger$ & $34 \dagger$ & $46 \dagger$ & $24 \dagger$ & $41+$ & $17 \dagger$ \\
\hline Histidine & 0 & 0 & 0 & 0 & 0 & 0 & 0 & 0 & $25 t$ & 0 & 0 & 0 \\
\hline Isoleucine & 68 & 88 & 83 & $80 \dagger$ & 97 & 98 & 74 & 77 & 101 & 97 & 109 & 103 \\
\hline Leucine & $64 \dagger$ & $73+$ & 94 & 93 & 95 & 94 & 0 & 0 & $9 \dagger$ & $7 \dagger$ & 0 & 0 \\
\hline Lysine & 88 & 101 & 113 & 115 & 92 & 94 & 83 & 88 & 97 & 97 & 110 & 113 \\
\hline Methionine & 76 & 73 & 94 & 106 & 95 & 93 & 81 & 85 & 106 & 99 & 108 & 114 \\
\hline Phenylalanine & 0 & $9 \dagger$ & $8+$ & $7 \dagger$ & 0 & 0 & 0 & 0 & $15 \dagger$ & $10 \dagger$ & 0 & 0 \\
\hline Threonine & 107 & 110 & 103 & 104 & 86 & 93 & 82 & 89 & 104 & 96 & 106 & 103 \\
\hline Tryptophan & 88 & 86 & 101 & 108 & 101 & 87 & 71 & 88 & 94 & 100 & 109 & 105 \\
\hline Tyrosine & 62 & 66 & 99 & 94 & 91 & 80 & 82 & 88 & 98 & 95 & 96 & 113 \\
\hline Valine & 0 & 0 & 0 & 0 & 0 & 0 & 0 & 0 & 0 & 0 & 0 & 0 \\
\hline
\end{tabular}

* Infection is expressed as a percentage of the number of infected cells in the control receiving complete medium.

† Inclusions were markedly smaller than those in the control receiving complete medium.

Table 3. Effect of single amino acid omissions on the growth of strains 434 and MRC-4f of C. trachomatis in cycloheximide-treated monolayers

Experimental conditions were as described in Table 1.

\begin{tabular}{|c|c|c|c|c|}
\hline \multirow{4}{*}{$\begin{array}{c}\text { Strain } \\
\text { Serotype } \\
\text { Omitted } \\
\text { amino acid }\end{array}$} & \multicolumn{4}{|c|}{ Infection* } \\
\hline & \multicolumn{2}{|c|}{$\begin{array}{l}434 \\
\mathrm{~L}_{2}\end{array}$} & \multicolumn{2}{|c|}{ MRC-4f } \\
\hline & & & & \\
\hline & Expt 1 & Expt 2 & Expt 1 & Expt 2 \\
\hline - & 100 & 100 & 100 & 100 \\
\hline Arginine & 97 & 111 & 103 & 97 \\
\hline Cysteine & 108 & 102 & 103 & 103 \\
\hline Glutamine & 0 & $9 \dagger$ & $10 \dagger$ & $4 \dagger$ \\
\hline Histidine & 0 & 0 & 0 & 0 \\
\hline Isoleucine & $77 \dagger$ & $90 \dagger$ & $88 \dagger$ & $53 \dagger$ \\
\hline Leucine & 0 & 0 & 0 & 0 \\
\hline Lysine & 88 & 110 & 101 & 96 \\
\hline Methionine & 0 & 0 & $53+$ & $16 \dagger$ \\
\hline Phenylalanine & 0 & 0 & $5+$ & $3+$ \\
\hline Threonine & 97 & 110 & 102 & 104 \\
\hline Tryptophan & 102 & 111 & 99 & 98 \\
\hline Tyrosine & 100 & 108 & 101 & 89 \\
\hline Valine & 0 & 0 & 0 & 0 \\
\hline
\end{tabular}

${ }^{*}$ Infection is expressed as a percentage of the number of infected cells in the control receiving complete medium.

$\dagger$ Inclusions were markedly smaller than those in the control receiving complete medium.

Effect of omission of single amino acids on the growth of C. psittaci strains

Omission of either leucine or valine completely inhibited the growth of all four $C$. psittaci strains (Table 4), whilst deletion of glutamine from the medium had a graded effect on chlamydial development. Growth of FKC was completely inhibited and that of EAE was largely prevented; inclusion formation by GP-IC and Cal-10 was reduced in terms of inclusion size but 
Table 4. Effect of single amino acid omissions on the growth of strains of C. psittaci in cycloheximide-treated monolayers

Experimental conditions were as described in Table 1, except that infected monolayers were fixed for examination at $26 \mathrm{~h}$ p.i. (GP-IC and Cal-10) or $48 \mathrm{~h}$ p.i. (EAE and FKC).

\begin{tabular}{|c|c|c|c|c|c|c|c|c|}
\hline \multirow{3}{*}{$\begin{array}{l}\text { Strain } \\
\text { Omitted } \\
\text { amino acid }\end{array}$} & \multicolumn{8}{|c|}{ Infection* } \\
\hline & \multicolumn{2}{|c|}{ GP-IC } & \multicolumn{2}{|c|}{$\mathrm{EAE}$} & \multicolumn{2}{|c|}{ Cal-10 } & \multicolumn{2}{|c|}{ FKC } \\
\hline & Expt 1 & Expt 2 & Expt 1 & Expt 2 & Expt 1 & Expt 2 & Expt 1 & Expt 2 \\
\hline$\ldots$ & 100 & 100 & 100 & 100 & 100 & 100 & 100 & 100 \\
\hline Arginine & 117 & 105 & 102 & 98 & 96 & 98 & 110 & 105 \\
\hline Cysteine & 105 & 110 & 96 & 94 & 95 & 91 & 107 & 98 \\
\hline Glutamine & $104+$ & $93 \dagger$ & $1 \dagger$ & $2 \dagger$ & $92 \dagger$ & $95 \dagger$ & 0 & 0 \\
\hline Histidine & 105 & 104 & 93 & 96 & 97 & 100 & 97 & 105 \\
\hline Isoleucine & 98 & 110 & 96 & 103 & 92 & 85 & 100 & 93 \\
\hline Leucine & 0 & 0 & 0 & 0 & 0 & 0 & 0 & 0 \\
\hline Lysine & 95 & 107 & 90 & 99 & 91 & 100 & 109 & 97 \\
\hline Methionine & 102 & 102 & 93 & 101 & $90 \dagger$ & $100 \dagger$ & 99 & 102 \\
\hline Phenylalanine & 0 & 0 & 0 & 0 & 0 & 0 & 104 & 92 \\
\hline Threonine & 100 & 106 & 94 & 103 & 100 & 94 & 106 & 104 \\
\hline Tryptophan & 95 & 103 & 93 & 99 & 86 & 76 & 99 & 96 \\
\hline Tyrosine & 113 & 79 & 0 & 0 & 92 & 99 & 103 & 104 \\
\hline Valine & 0 & 0 & 0 & 0 & 0 & 0 & 0 & 0 \\
\hline
\end{tabular}

not in numbers of infected cells. Among the four strains, EAE showed a unique requirement for provision of tyrosine, and FKC could be distinguished by its lack of requirement for supplied phenylalanine.

The amino acid requirements of GP-IC and Cal-10 were very similar; Cal-10 differed only in showing a reduction in inclusion size in the absence of supplied methionine.

\section{Range of amino acid requirements for growth of 15 strains of chlamydiae}

Of the 13 amino acids tested there was no requirement for four of these: arginine, cysteine, lysine and threonine; all other amino acids were required by one or more strains. Glutamine, leucine and phenylalanine were required by the majority of strains tested and valine was required by all. Histidine was required by all of the $C$. trachomatis strains tested but by none of the four $C$. psittaci strains.

\section{DISCUSSION}

The pattern of requirements for supplied amino acids examined for 11 C. trachomatis and four C. psittaci strains suggests that the two species can be distinguished from one another and that, within $C$. trachomatis, serotypes can be grouped according to the clinical syndrome with which they are commonly associated. The four $C$. psittaci strains were chosen to reflect different natural hosts, and the observations of three distinct patterns of requirement suggests that they may correlate with host origin. These conclusions remain tentative until confirmation by analysis of further isolates and examination of the effects of amino acid deprivation upon infectivity yield, as for GP-IC (Allan \& Pearce, 1983). The latter is desirable since, for some strains, absence of amino acid may lead to inclusions which, although normal in size by microscopic observation, have a high content of reticulate bodies consequent upon arrest of development.

Our interpretation of the phenomena is based on the previous work with strain GP-IC (Allan \& Pearce, 1983). Upon deprivation of certain amino acids, inhibition of growth appears to occur, not because host cell catabolism is unable quantitatively to fulfil chlamydial requirements, but because provision fails to exceed a certain threshold concentration - the latter 
being defined as the concentration required for an infectivity and organism yield equal to that observed in fully supplemented medium. Absence of some amino acids resulted in reduced inclusion size rather than complete inhibition of growth. A similar effect was observed for GPIC below threshold levels of phenylalanine or valine (Allan \& Pearce, 1983). It may be, therefore, that the effect is manifested when host cell provision alone, approaches, but does not quite reach, the threshold requirement. It may, on the other hand, arise for amino acids for which there is no threshold and for which reduction in extracellular concentration leads to progressively lower yields of organisms. The finding that in some instances amino acid deletion resulted in intermediate and variable numbers of infected cells is less easy to explain. It may simply be due to heterogeneity in the nutritional contents of host cells, or alternatively, heterogeneity in the chlamydial population in its ability to utilize available amino acid. The effect appeared most frequently following glutamine deletion from medium (Tables 1, 2, 3 and 4) and, as suggested for reduction in inclusion size, may occur when host cell provision approaches chlamydial growth requirements. Glutamine is utilized in a number of biosynthetic pathways and it is notable that, of the amino acids analysed in spent medium, it was the only one showing a marked and consistent reduction when medium from uninfected cells was analysed and compared with that from $10 \%$ and $95 \%$ GP-IC-infected cell monolayers (Allan \& Pearce, 1983).

It should be emphasized that the data presented do not provide an analysis of the complete amino acid requirements of any chlamydial strain. They pinpoint only those amino acids for which omission of one in the presence of 12 others at their normal concentrations results in growth inhibition, as previously discussed (Allan \& Pearce, 1983). Examination of further isolates within the $C$. trachomatis oculogenital group (serotypes D-I) may allow subdivision on the basis of differing requirements for leucine, and possibly isoleucine, methionine and tyrosine (see Table 2). But such more detailed biotyping might best be done on rinsed or starved cells, to reduce host amino acid pools, together with titration of threshold concentrations for growth. Using host cells pre-starved for $60 \mathrm{~h}$, Karayiannis \& Hobson (1981) identified cysteine/cystine, isoleucine and tyrosine as essential for growth of a $C$. trachomatis strain, serotype $\mathrm{D} / \mathrm{E}$, in addition to those amino acids described here for serotypes D-I.

The amino acid requirements of serotypes $L_{1}$ and $L_{3}$, in the LGV group, have not been examined. However, the relative reproducibility of the patterns so far observed, and their segregation into groups according to clinical syndrome, would suggest that $L_{1}$ and $L_{3}$ isolates should be similar, if not identical to $L_{2}$ in requirements. On the same grounds, it seems reasonable to expect that examples of serotypes $\mathbf{J}$ and $\mathrm{K}$ (oculogenital group) should not greatly differ from serotypes D-I. It may be of interest to test whether $C$. trachomatis strains isolated from cases of infant pneumonia (Schachter \& Grossman, 1981) deviate from the patterns reported here. The similarity of LGV strain 434 and the 'fast' strain, MRC-4f (Table 3), supports the hypothesis that variant 'fast' strains share a common origin with LGV strains (Wang \& Grayston, $1971 b$ ). The amino acid requirements of MRC-4f and MRC-4 (originally believed to be the parent strain; serotype E, Table 2) differed appreciably. These results are in agreement with the microimmunofluorescence and mouse toxicity prevention tests of Wang \& Grayston $(1971 b)$ who pointed out that, at that time, methods were not readily available for purification of mixed cultures of chlamydiae originating from clinical material. The data presented here may permit selective inhibition of LGV in the presence of other $C$. trachomatis strains - a particular problem because of other features of LGV strains which make them highly infective in cell culture. Appropriate amino acid deletions should also inhibit trachoma in the presence of non-trachoma, and $C$. trachomatis in the presence of $C$. psittaci strains.

The finding of three clear patterns of amino acid requirement for the four C. psittaci strains examined suggests that this form of biotyping may be a useful means of classifying strains within the $C$. psittaci species. Of particular interest would be to determine whether strains capable of producing psittacosis in man carry a specific, coincidental marker of nutritional demand which could provide a rapid indication of the possible pathogenicity for man of clinical isolates of $C$. psittaci. The only distinguishing feature between Cal-10 and GP-IC was a reduction in the size of the inclusions produced by $\mathrm{Cal}-10$ in the absence of supplied methionine. The similarity in nutritional requirements suggests that the two parasites may have evolved along similar routes. To a certain extent this is supported by the observation that the guinea pig pathogen, GP-IC, is 
capable of growth in the conjunctiva of both the guinea pig and the ferret. However, infection of the heterologous host occurred only for GP-IC; Cal-10 grew in the ferret conjunctiva but not in that of the guinea pig (I. Allan and J. H. Pearce, unpublished).

The pattern of amino acid requirements seen overall emphasizes the near universal need for phenylalanine, the branched chain amino acids leucine and valine, and histidine (for C. trachomatis strains). It has been suggested that $C$. psittaci may synthesize histidine (Moulder, 1966), but how far relief from requirement for leucine (see Table 2) or phenylalanine (FKC; Table 4) results from biosynthesis is not known. Speculation on the possible significance of these effects for host infection is best deferred until we have information on how growth is regulated by amino acid deprivation (Allan \& Pearce, 1983). However, it may not be coincidental that branched chain amino acids, especially leucine, have a marked influence on protein synthesis in mammalian and bacterial systems in conditions of nutrient limitation (Tischler et al., 1982; Quay \& Oxender, 1980).

We are indebted for gifts of strains to Drs S. Darougar, S. J. Richmond, J. D. Treharne, R. M. Woodland and P. B. Wyrick, and to Dr P. Brown for providing facilities for work with feline keratoconjunctivitis. We thank the Medical Research Council for financial support.

\section{REFERENCES}

Allan, I. \& Pearce, J. H. (1983). Differential amino acid utilization by Chlamydia psittaci (strain guinea pig inclusion conjunctivitis) and its regulatory effect on chlamydial growth. Journal of General Microbiology 129, 1991-2000.

Collier, L. H. \& SMItH, A. (1967). Dissemination and immunogenicity of live TRIC agent in baboons after parenteral injection. American Journal of Ophthalmology 63, 1589-1602.

Darougar, S., MonNickendam, M. A., El-SheikH, H., Treharne, J. D., Woodland, R. M. \& Jones, B. R. (1977). Animal models for the study of chlamydial infections of the eye and genital tract. In Nongonococcal Urethritis and Related Infections, pp. 186-198. Edited by K. K. Holmes \& D. Hobson. Washington: American Society for Microbiology.

Francis, T. \& Magill, T. P. (1938). An unidentified virus producing acute meningitis and pneumonitis in experimental animals. Journal of Experimental Medicine 68, 147-160.

Griffiths, M. S., Ainsworth, S. \& Pearce, J. H. (1976). Infectivity titration of guinea-pig inclusion conjunctivitis agent in irradiated McCoy cells. Journal of General Microbiology 95, 249-256.

HowIE, J. (Chairman) (1978). Code of Practice for Prevention of Infection in Clinical Laboratories and Postmortem Rooms. London: Her Majesty's Stationery Office.

Karayiannis, P. \& Hobson, D. (1981). Amino acid requirements of a Chlamydia trachomatis genital strain in McCoy cell cultures. Journal of Clinical Microbiology 13, 427-432.

Kuo, C. C., Wang, S. P. \& Grayston, J. T. (1977). Growth of trachoma organisms in HeLa-229 cell cultures. In Nongonoccal Urethritis and Related Infections, pp. 328-336. Edited by K. K. Holmes \& D. Hobson. Washington: American Society for Microbiology.

MouldER, J. W. (1966). The relation of the psittacosis group (chlamydiae) to bacteria and viruses. Annual Review of Microbiology 20, 107-130.

MouldeR, J. W. (1974). Intracellular parasitism: life in an extreme environment. Journal of Infectious Diseases 130, 300-306.

MurRay, E. S. (1964). Guinea pig inclusion conjunctivitis virus. I. Isolation and identification as a member of the psittacosis-lymphogranulomatrachoma group. Journal of Infectious Diseases 114, $1-12$.

QuAY, S. C. \& OXENDER, D. L. (1980). Regulation of membrane transport. In Biological Regulation and Development, vol. 2, pp. 413-436. Edited by R. F. Goldberger. New York: Plenum Press.

Reeve, P. \& Taverne, J. (1963). Observations on the growth of trachoma and inclusion blennorrhoea viruses in embryonate eggs. Journal of Hygiene 61, $67-75$.

Schachter, J. \& Grossman, M. (1981). Chlamydial infections. Annual Review of Medicine 32, 45-61.

Stamp, J. T., McEwen, A. D., WatT, J. A. A. \& Nisbet, D. J. (1950). Enzootic abortion in ewes. I. Transmission of the disease. Veterinary Record 62, 251-254.

Tischler, M. E., Desautels, M. \& Goldberg, A. L. (1982). Does leucine, leucyl-tRNA, or some metabolite of leucine regulate protein synthesis and degradation in skeletal and cardiac muscle? Journal of Biological Chemistry 257, 1613-1621.

Treharne, J. D., Dines, R. J. \& Darougar, S. (1977). Serological responses to chlamydial ocular and genital infections in the United Kingdom and Middle East. In Nongonococcal Urethritis and Related Infections, pp. 249-258. Edited by K. K. Holmes \& D. Hobson. Washington: American Society for Microbiology.

WANG, S. P. \& Grayston, J. T. (1971a). Classification of TRIC and related strains with micro-immunofluorescence. In Trachoma and Related Disorders, pp. 305-321. Edited by R. L. Nichols. Amsterdam: Excerpta Medica.

WANG, S. P. \& Grayston, J. T. (1971 b). Studies on the identity of the 'fast' egg-killing chlamydia strains. In Trachoma and Related Disorders, pp. 322-336. Edited by R. L. Nichols. Amsterdam: Excerpta Medica. 